\title{
Adipose tissue, estradiol levels, and bone health in obese men with metabolic syndrome
}

\author{
Marie Juul Ornstrup ${ }^{1,2}$, Thomas Nordstrøm Kjær ${ }^{1}$, Torben Harsløf', \\ Hans Stødkilde-Jørgensen ${ }^{3}$, David Michael Hougaard ${ }^{4}$, Arieh Cohen ${ }^{4}$, \\ Steen Bønløkke Pedersen ${ }^{1,2}$ and Bente Lomholt Langdahl ${ }^{1,2}$
}

'Department of Endocrinology, Aarhus University Hospital, Tage-Hansens Gade 2, Entrance 3C, DK-8000 Aarhus C, Denmark, ${ }^{2}$ Department of Clinical Medicine, Aarhus University, Nordre Ringgade 1, DK-8000 Aarhus, Denmark,

${ }^{3}$ The MR Research Center, Aarhus University Hospital, Skejby, Brendstrupgaardsvej 100, DK-8200 Aarhus N, Denmark and ${ }^{4}$ Department of Clinical Biochemistry, Immunology and Genetics, Statens Serum Institut, Artillerivej 5, DK-2300 Copenhagen S, Denmark

\author{
Correspondence \\ should be addressed \\ to M J Ornstrup \\ Email \\ marie.juul.ornstrup@ \\ clin.au.dk
}

\section{Abstract}

Objective: Visceral adipose tissue (VAT) is associated with an increased risk of metabolic syndrome (MetS). Recent studies have suggested that VAT negatively affects bone. However, MetS has also been associated with higher estradiol ( $\left.E_{2}\right)$ levels, which is bone protective. We therefore investigated the impact of VAT and $E_{2}$ levels on bone density, structural parameters, and strength estimates.

Design: A cross-sectional study was conducted in 72 obese men with MetS to investigate the impact of VAT and $E_{2}$ levels on bone. Methods: Bone parameters were assessed by dual-energy X-ray absorptiometry (DXA), quantitative computed tomography (QCT), and high-resolution peripheral QCT (HRpQCT) at lumbar spine, proximal femur, radius, and tibia. VAT volume was measured by magnetic resonance imaging (MRI) and sexual hormones were measured in blood samples.

Results: Men with high VAT had a lower bone density at the hip $(P<0.05)$, lower cortical thickness, and higher buckling ratio at femoral neck (FN) $(P=0.008$ and $P=0.02)$, compared with men with low VAT, despite a similar body weight and BMI. Generally, $E_{2}$ levels were low (median 43 pmol/l), and men with $E_{2}$ levels below median had reduced bone density at lumbar spine $(P=0.04)$, and impaired structural parameters at radius and tibia, compared with men with $\mathrm{E}_{2}$ levels above median. At the hip, VAT volume and $E_{2}$ levels affected bone density independently and additively, and $50 \%$ of men with high VAT and low $E_{2}$ levels had osteopenia with significantly lower $T$-score at FN $(P=0.004)$.

Conclusions: High VAT and low $E_{2}$ negatively affect bone in obese men with MetS. VAT and $E_{2}$ affect bone density at the hip independently and additively, revealing an unexpected high prevalence of osteopenia in middle-aged men with MetS.

\section{Introduction}

The prevalence of obesity and osteoporosis has rapidly increased over the last decades $(1,2)$. The bone-fat relationship has proven to be complex and published results in the field have been ambiguous. Body weight and BMI are positively correlated with bone mineral density (BMD) $(3,4,5)$. However, obese individuals seem to have increased fracture risk regardless of a higher $\operatorname{BMD}(6,7,8)$,
(C) 2015 European Society of Endocrinology Printed in Great Britain and similarly for patients with type 2 diabetes $(9,10,11)$. Obesity, metabolic syndrome (MetS), and type 2 diabetes are all associated with low-grade inflammation $(12,13)$. As visceral adipose tissue (VAT) produces inflammatory cytokines that are harmful to bone, research has focused on the role of VAT on bone $(14,15,16,17)$. At odds with this, a large VAT depot is also associated with an

Published by Bioscientifica Ltd. 
increased aromatase activity and increased levels of estradiol $\left(\mathrm{E}_{2}\right)(18,19)$, which is bone protective $(20)$. Khosla et al. (21) found no association between bone density and sex steroid levels in eugonadal young men, but did find positive associations between bone density and low testosterone and $\mathrm{E}_{2}$ levels in elderly men.

The effect of VAT on bone has previously been investigated in a number of studies. Gilsanz et al. (22) showed that, in young women, VAT was negatively correlated with BMD, whereas subcutaneous adipose tissue (SAT) was positively correlated with BMD. Yamaguchi et al. (23) showed that, in men with type 2 diabetes, both SAT and VAT were positively correlated with BMD. When data were adjusted for body weight, opposing results emerged, but VAT remained inversely related to the risk of vertebral fractures. Finally, Choi et al. (24) found that VAT was negatively correlated with BMD, whereas SAT was positively correlated with BMD in Korean men and women, after adjusting for body weight. These seemingly conflicting results may be due to the fact that SAT, VAT, waist circumference (WC), and BMI are highly correlated in most populations $(25,26)$ and this may potentially disturb statistical analyses due to collinearity.

We therefore investigated the impact of visceral fat volume and serum levels of $\mathrm{E}_{2}$ on areal BMD (aBMD), volumetric BMD (vBMD), bone structure, and estimates of bone strength in a very homogeneous population of obese middle-aged men with MetS.

\section{Subjects and methods}

\section{Study design and subjects}

This was a cross-sectional study on baseline data from a randomized, placebo-controlled, double-blinded, singlecenter study (27). Seventy-two subjects were included in this study. Inclusion criteria were male sex, age between 30 and 60 years, and MetS. MetS was defined according to the International Diabetes Federation (28) as central obesity (WC $\geq 94 \mathrm{~cm}$ and/or BMI $>30 \mathrm{~kg} / \mathrm{m}^{2}$ ) plus any two of the following: raised triglycerides $(\geq 1.7 \mathrm{mmol} / \mathrm{l})$, reduced $\mathrm{HDL}(\leq 1.03 \mathrm{mmol} / \mathrm{l})$, raised blood pressure (systolic $\geq 130 \mathrm{mmHg}$ or diastolic $\geq 85 \mathrm{mmHg}$ ), raised fasting plasma glucose $(\geq 5.6 \mathrm{mmol} / \mathrm{l})$, or drug treatment for the individual features. Exclusion criteria were other overt endocrine diseases, renal disease, hepatic disease, heart disease, malignant disease, anemia, alcohol abuse, planned lifestyle changes, and absolute or relative contraindications for magnetic resonance imaging (MRI).

\section{Ethical aspects}

The protocol was approved by the Regional Committee on Health Research Ethics (M-20110111) and the Danish Data Protection Agency, and the study was conducted in agreement with the Declaration of Helsinki II. Participants were given oral and written information about the purpose and nature of all procedures before informed consent was obtained. The protocol was registered at clinicaltrials.gov (NCT01412645) before recruitment was initiated.

\section{Magnetic resonance imaging}

VAT and SAT were determined with MRI using a fast spin echo sequence (Signa Excite 1.5 Tesla, Twin Speed; GE Medical Systems, Little Chalfont, Bucks, UK). Axial slices were obtained from the proximal border of the femoral heads to the upper pole of the most superior kidney, and Hippo Fat was used for data processing, providing estimates for SAT and $\operatorname{VAT}(29,30)$.

\section{Dual-energy X-ray absorptiometry}

aBMD at the lumbar spine (L1-L4) and hip was measured by dual-energy X-ray absorptiometry (DXA) using a Hologic Discovery scanner. Coefficient of variation $(\mathrm{CV})$ of repositioning is $1.5 \%$ for lumbar spine BMD and $2.1 \%$ for femoral neck (FN) $(31,32)$. In addition, whole-body fat mass and lean body mass were measured by DXA.

\section{Quantitative computed tomography}

vBMD at lumbar vertebra 2 (LS vBMD) and the proximal femur was measured using a Philips Brilliance 40 multidetector helical CT scanner. CT scans were acquired from the distal endplate of L1 to the proximal endplate of L3, and from acetabulum directly above the femoral head to $2 \mathrm{~cm}$ below the lesser trochanter (TR), with $3 \mathrm{~mm}$ slice thickness and spacing as described previously (27). M J Ornstrup analyzed all quantitative computed tomography (QCT) data, blinded to VAT distribution and $\mathrm{E}_{2}$ levels, with a reanalysis precision of $0.6 \pm 0.6 \%$ for lumbar spine (LS) $\mathrm{vBMD}_{\text {trab}}$, and $0.6 \pm 0.7 \%$ for total hip $(\mathrm{TH})$ vBMD integral

We determined $\mathrm{vBMD}_{\text {integral }}$ in standard regions $(\mathrm{TH}$, FN, TR, and intertrochanteric (IT)), along with separate trabecular estimates $\left(\mathrm{vBMD}_{\text {trab}}\right)$ and cortical estimates $\left(\mathrm{vBMD}_{\text {cortical }}\right)$. A fixed threshold for cortical separation of $0.350 \mathrm{~g} / \mathrm{cm}^{3}$ was used. Geometric measures at the FN; 
cross-sectional area (CSA) total, CSA $_{\text {cortical, and cortical }}$ thickness (Ct.Th), along with estimates of bone strength (cross-sectional moment of inertia (CSMI) and buckling ratio) were determined $(33,34,35)$.

\section{High-resolution peripheral QCT}

Assessment of geometry and microarchitecture of the non-dominant distal radius and tibia (or in case of a previous fracture in the non-fractured limb) were obtained using high-resolution peripheral QCT (HRpQCT) (XtremeCT, Scanco Medical, Brüttisellen, Switzerland) as described previously (27). The analysis was performed by M J Ornstrup, who was blinded to VAT distribution and $\mathrm{E}_{2}$ levels. $\mathrm{CV}$ of repositioning for radius and tibia density measures were $0.5-1.5 \%$ and the structural parameters $1-5 \%(36,37)$. The following parameters were measured or calculated: trabecular bone volume fraction $(\mathrm{BV} / \mathrm{TV})$ is derived from the trabecular volume density, assuming a mineral density of fully mineralized bone of $1200 \mathrm{mg}$ hydroxyapatite $/ \mathrm{cm}^{3}$. Trabecular number (Tb.N) is a direct measure. Trabecular thickness (Tb.Th) and trabecular space (Tb.Sp) are calculated from Tb.N and BV/TV (38).

\section{Biochemistry}

We analyzed bone alkaline phosphatase (BAP), osteoprotegerin (OPG), osteocalcin (intact and N-terminal fragment), and procollagen 1 N-terminal propeptide (P1NP) as markers of bone formation. As markers of bone resorption, we analyzed C-terminal telopeptide of type 1 collagen (CTx), and cross-linked N-terminal telopeptide of type 1 collagen (NTx). In addition, parathyroid hormone (PTH) and 25-hydroxy vitamin D were measured. All analyses were performed by standard laboratory methods at the Department of Clinical Biochemistry (Aarhus University Hospital, Denmark), except OPG. OPG was measured using ELISA OPG Kit (Biomedica, Vienna, Austria). Intra- and inter-assay $\mathrm{CV}$ values were 4 and 5\% respectively.

Markers reflecting metabolic state were measured; triglyceride, HDL, and alanine transaminase (ALT) were performed by standard laboratory methods at the Department of Clinical Biochemistry (Aarhus University Hospital, Denmark). Interleukin 6 (IL6), high-sensitivity C-reactive protein (hs-CRP), leptin, and adiponectin were analyzed using ELISA as described previously (39), and glucose using ELISA Kit from Roche with intra- and interassay CV values of 3 and 4\% respectively. Sex steroid hormones ( $\mathrm{E}_{2}$, estrone, testosterone, androstenedione, and sex hormone-binding globulin (SHBG)) were measured by standard laboratory methods at the Danish National Health Laboratory.

\section{Statistical analysis}

Normality was checked by QQ-plots, and test for equal variance between groups was assessed by Levene's test for equal variances. The level of significance was 0.05. Analyses and graphs were performed with IBM SPSS Statistics (version 20), and SigmaPlot (version 12.5). Correlations between two variables were assessed by the Spearman's rank-order correlation. To determine the effect of VAT and $\mathrm{E}_{2}$ on vBMD, structural bone parameters, and strength estimates, the study subjects were divided into two groups based on VAT median and $\mathrm{E}_{2}$ median. Men with VAT below the median were grouped in $\mathrm{VAT}_{\text {low, }}$, and men with VAT above the median were grouped in $\mathrm{VAT}_{\text {high }}$. Additionally, study subjects were divided into $\mathrm{E}_{2 \text { low }}$ and $\mathrm{E}_{2 \mathrm{high}}$ according to $\mathrm{E}_{2}$ levels below and above $\mathrm{E}_{2}$ median.

To adjust for multiple comparisons, the multivariate $t$-test (Hotelling's $t$-test) was performed on biochemical markers of bone turnover and QCT-derived hip parameters. Variable dependency is accounted for within this multivariate test. Thereafter, differences between VAT groups and $\mathrm{E}_{2}$ groups, respectively, were assessed by unpaired Student's t-test, or the Mann-Whitney rank sum test. Results are presented as mean \pm s.D. or as median with interquartile (25\%; 75\%) range. Multiple linear regression analysis was used to investigate whether VAT group and $\mathrm{E}_{2}$ group had independent impact on bone density at the hip. Finally, the population was divided according to VAT volume and $\mathrm{E}_{2}$ levels into four groups $\left(\mathrm{E}_{2 \text { low }}+\mathrm{VAT}_{\mathrm{high}}\right.$, $\mathrm{E}_{2 \text { low }}+\mathrm{VAT}_{\text {low }}, \mathrm{E}_{2 \text { high }}+\mathrm{VAT}_{\text {high, }}$ and $\left.\mathrm{E}_{2 \text { high }}+\mathrm{VAT}_{\text {low }}\right)$, and ANOVA with Student-Newman-Keuls post hoc adjustment was used to assess differences between groups.

\section{Results}

\section{Population characteristics}

The population consisted of 72 men aged $49.2 \pm 6.3$ years. Baseline characteristics relevant to MetS were as follows: BMI of $33.6 \pm 3.3 \mathrm{~kg} / \mathrm{m}^{2}$, WC $114(108 ; 119) \mathrm{cm}$, triglyceride $1.8(1.3 ; 2.3) \mathrm{mmol} / \mathrm{l}$, HDL $1.2(1.0 ; 1.4) \mathrm{mmol} / \mathrm{l}$, systolic blood pressure $145.5 \pm 15.5 \mathrm{mmHg}$, diastolic blood pressure $89.1 \pm 9.9 \mathrm{mmHg}$, and fasting plasma glucose 5.8 $(5.3 ; 6.0) \mathrm{mmol} / \mathrm{l}$. In this homogeneous population, BMI and SAT (Fig. 1A) and WC and SAT (Fig. 1B) were positively correlated, but no correlations were found between BMI and VAT (Fig. 1C) or WC and VAT (Fig. 1D). 

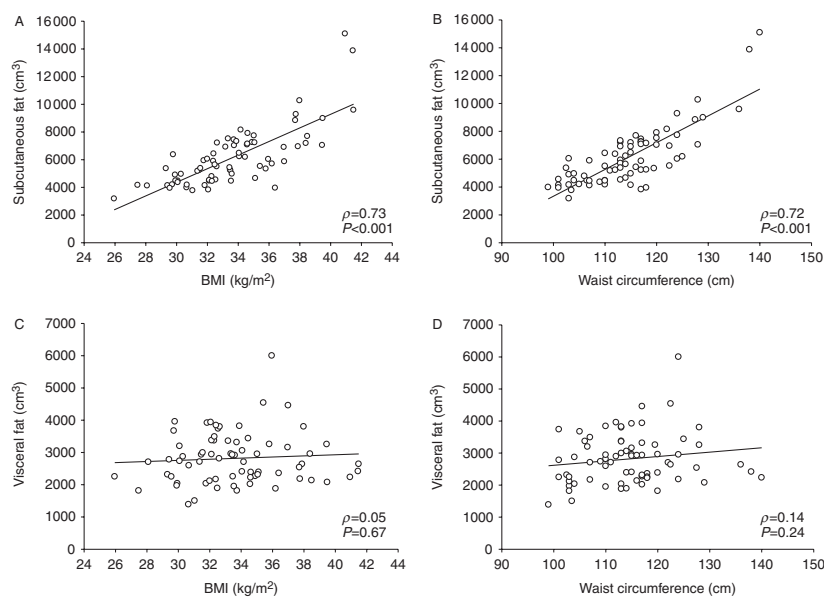

\section{Figure 1}

Correlations between BMI and SAT (A), WC and SAT (B), BMI and VAT (C), and WC and VAT (D). Open circle represents each trial subject $(n=72) . \rho$ and $P$ value from the Spearman's rank-order correlation.

\section{Clinical and metabolic characteristics across visceral fat mass median}

Median VAT for $\mathrm{VAT}_{\text {low }}$ and $\mathrm{VAT}_{\text {high }}$ groups were 2239 and $3283 \mathrm{~cm}^{3}$ respectively $(P<0.001)$. Clinical characteristics such as age, weight, BMI, and WC did not differ between groups (Table 1). Other measures of fat distribution, including whole-body fat mass (by DXA), and subcutaneous fat mass (MRI) did not differ between groups either (Table 1). The level of ALT was significantly higher in the $\mathrm{VAT}_{\text {high }}$ group, IL6 borderline was significantly higher, and HDL was significantly lower in the $\mathrm{VAT}_{\text {high }}$ group (Table 1). Other metabolic markers, including insulin, adiponectin, and leptin, were not different between groups (Table 1).

\section{Bone density and structural parameters across visceral fat median}

$\mathrm{vBMD}_{\text {integral }}$ at $\mathrm{TH}$ was significantly lower in the $\mathrm{VAT}_{\text {high }}$ group compared with the $\mathrm{VAT}_{\text {low }}$ group $(P=0.01)$. Similar results were found at $\mathrm{FN}, \mathrm{TR}$, and IT, although only borderline significant at TR (Table 2). In addition, the subanalysis of $\mathrm{vBMD}_{\text {trab }}$ at $\mathrm{TH}$ was significantly lower in the VAT $_{\text {high }}$ group $(P=0.02)$, with similar site-specific effects at FN, TR, and IT (Table 2). vBMD cortical $_{\text {at }} \mathrm{TH}$ was higher in the $\mathrm{VAT}_{\text {high }}$ group compared with the $\mathrm{VAT}_{\text {low }}$ group $(P=0.01)$, and similarly at IT. At FN and TR, vBMD ${ }_{\text {cortical }}$ did not differ between groups (Table 2). $\mathrm{CSA}_{\text {total }}$ at FN was similar across VAT groups (Table 2), while FN CSA cortical and $\mathrm{Ct}$.Th were significantly lower in the $\mathrm{VAT}_{\text {high }}$ group compared with the $\mathrm{VAT}_{\text {low }}$ group $(P=0.005$ and $P=0.008$ respectively). Furthermore, FN buckling ratio was significantly higher in the $\mathrm{VAT}_{\text {high }}$ group compared with the $\mathrm{VAT}_{\text {low }}$ group $(P=0.02)$. Multivariate $t$-test on all the above-mentioned QCT-derived hip parameters across VAT groups revealed overall significant effects $(P=0.027)$.

Table 1 Clinical and metabolic features across visceral fat median. Data are expressed as mean \pm s.D. or median with interquartile range $(25 \% ; 75 \%)$.

\begin{tabular}{l}
\hline \\
\hline Visceral fat $\left(\mathrm{cm}^{3}\right)$ \\
Age (years) \\
Weight $(\mathrm{kg})$ \\
BMI $\left(\mathrm{kg} / \mathrm{m}^{2}\right)$ \\
Waist circumference $(\mathrm{cm})$ \\
Whole-body fat mass $(\mathrm{kg})$ \\
Subcutaneous fat $\left(\mathrm{cm}^{3}\right)$ \\
ALT $(\mathrm{U} / \mathrm{l})$ \\
Systolic blood pressure $(\mathrm{mmHg})$ \\
Diastolic blood pressure $(\mathrm{mmHg})$ \\
Glucose $(\mathrm{mmol} / \mathrm{l})$ \\
HDL (mmol/l) \\
Triglyceride $(\mathrm{mmol} / \mathrm{l})$ \\
IL6 (pg/ml) \\
hs-CRP $(\mathrm{mg} / \mathrm{l})$ \\
Leptin $(\mathrm{ng} / \mathrm{ml})$ \\
Adiponectin $(\mathrm{ng} / \mathrm{ml})$ \\
Insulin $(\mathrm{pmol} / \mathrm{l})$
\end{tabular}

\begin{tabular}{c}
\hline $\mathbf{V A T}_{\text {low }}(n=36)$ \\
\hline $2239(2021 ; 2400)$ \\
$48.3 \pm 6.3$ \\
$107.7(97.7 ; 117.7)$ \\
$33.9(30.8 ; 36.3)$ \\
$115(105 ; 120)$ \\
$32.9(27.9 ; 39.3)$ \\
$5917(4462 ; 7580)$ \\
$35(30 ; 43)$ \\
$143 \pm 14$ \\
$88 \pm 9$ \\
$5.65(5.30 ; 6.15)$ \\
$1.25(1.10 ; 1.40)$ \\
$1.60(1.10 ; 2.30)$ \\
$1.12(0.70 ; 1.78)$ \\
$2.60(1.45 ; 4.80)$ \\
$16.1(11.3 ; 20.9)$ \\
$1.41 \pm 0.58$ \\
$103.2(54.6 ; 129.0)$
\end{tabular}

\begin{tabular}{ccc} 
VAT $_{\text {high }}(n=36)$ & & $P$ value \\
\cline { 1 - 1 } $3283(2941 ; 3798)$ & & $<0.001$ \\
$50.1 \pm 6.2$ & & 0.22 \\
$107.6(103.5 ; 114.3)$ & & 0.83 \\
$32.9(31.9 ; 34.9)$ & & 0.57 \\
$114(110 ; 117)$ & & 0.88 \\
$33.7(31.4 ; 37.1)$ & & 0.34 \\
$5466(4530 ; 6915)$ & & 0.48 \\
$43(35 ; 55)$ & & 0.04 \\
$148 \pm 17$ & & 0.13 \\
$90 \pm 11$ & & 0.36 \\
$5.87(5.30 ; 6.01)$ & & 0.75 \\
$1.10(0.95 ; 1.35)$ & & 0.04 \\
$1.90(1.43 ; 2.38)$ & & 0.13 \\
$1.54(1.07 ; 2.20)$ & & 0.07 \\
$2.75(1.90 ; 5.20)$ & 0.56 \\
$14.3(12.4 ; 17.8)$ & 0.62 \\
$1.33 \pm 0.45$ & & 0.57 \\
$83.9(64.8 ; 100.4)$ & 0.45
\end{tabular}

The $P$ values were calculated by unpaired Student's $t$-test or the Mann-Whitney rank sum test, where appropriate. Significant results are highlighted in bold. VAT low, visceral adipose tissue volume below median VAT; VAT $_{\text {high, }}$ visceral adipose tissue volume above median VAT; ALT, alanine transaminase; IL6, interleukin 6; hs-CRP, high-sensitivity C-reactive protein. 
Table 2 Bone characteristics across visceral fat median. Data are expressed as mean \pm s.D. or median with interquartile range $(25 \% ; 75 \%)$.

\begin{tabular}{|c|c|c|c|}
\hline & $\operatorname{VAT}_{\text {low }}(n=36)$ & $\operatorname{VAT}_{\text {high }}(n=36)$ & $P$ value \\
\hline Lumbar spine $v B M D\left(\mathrm{~g} / \mathrm{cm}^{3}\right)$ & $0.149 \pm 0.03$ & $0.140 \pm 0.03$ & 0.22 \\
\hline \multicolumn{4}{|l|}{ Hip vBMD $\left(\mathrm{g} / \mathrm{cm}^{3}\right)$} \\
\hline \multicolumn{4}{|l|}{ Total hip } \\
\hline $\mathrm{VBMD}_{\text {integral }}$ & $0.330 \pm 0.05$ & $0.303 \pm 0.04$ & 0.01 \\
\hline $\mathrm{vBMD}_{\text {trab }}$ & $0.170 \pm 0.02$ & $0.158 \pm 0.02$ & 0.02 \\
\hline $\mathrm{vBMD}_{\text {cortical }}$ & $0.811 \pm 0.04$ & $0.834 \pm 0.04$ & 0.01 \\
\hline \multicolumn{4}{|l|}{ Femoral neck } \\
\hline $\mathrm{vBMD}_{\text {integral }}$ & $0.315 \pm 0.05$ & $0.283 \pm 0.04$ & 0.003 \\
\hline $\mathrm{vBMD}_{\text {trab }}$ & $0.191 \pm 0.02$ & $0.178 \pm 0.03$ & 0.03 \\
\hline $\mathrm{vBMD}_{\text {cortical }}$ & $0.604 \pm 0.03$ & $0.598 \pm 0.04$ & 0.47 \\
\hline \multicolumn{4}{|l|}{ Trochanter } \\
\hline $\mathrm{vBMD}_{\text {integral }}$ & $0.248 \pm 0.04$ & $0.233 \pm 0.03$ & 0.08 \\
\hline $\mathrm{vBMD}_{\text {trab }}$ & $0.164 \pm 0.02$ & $0.154 \pm 0.02$ & 0.05 \\
\hline $\mathrm{vBMD}_{\text {cortical }}$ & $0.750(0.72 ; 0.77)$ & $0.770(0.73 ; 0.82)$ & 0.12 \\
\hline \multicolumn{4}{|l|}{ Intertrochanteric } \\
\hline vBMD $_{\text {integral }}$ & $0.387 \pm 0.06$ & $0.354 \pm 0.05$ & 0.01 \\
\hline $\mathrm{vBMD}_{\text {trab }}$ & $0.174 \pm 0.02$ & $0.160 \pm 0.03$ & 0.02 \\
\hline $\mathrm{VBMD}_{\text {cortical }}$ & $0.820(0.81 ; 0.85)$ & $0.850(0.82 ; 0.87)$ & 0.01 \\
\hline \multicolumn{4}{|c|}{ Femoral neck geometry and strength } \\
\hline $\mathrm{CSA}_{\text {total }}\left(\mathrm{cm}^{2}\right)$ & $11.75 \pm 1.40$ & $11.91 \pm 0.25$ & 0.65 \\
\hline $\mathrm{CSA}_{\text {cortical }}\left(\mathrm{cm}^{2}\right)$ & $3.23(2.93 ; 3.93)$ & $2.85(2.52 ; 3.30)$ & 0.005 \\
\hline Cortical thickness $(\mathrm{mm})$ & $0.305(0.24 ; 0.36)$ & $0.261(0.22 ; 0.29)$ & 0.008 \\
\hline $\operatorname{CSMI}\left(\mathrm{mm}^{4}\right)$ & $7.21 \pm 1.56$ & $6.82 \pm 1.60$ & 0.30 \\
\hline Buckling ratio (1) & $6.17(5.05 ; 8.53)$ & $7.48(6.47 ; 9.16)$ & 0.02 \\
\hline \multicolumn{4}{|l|}{ Radius geometry } \\
\hline $\mathrm{BV} / \mathrm{TV}(1)$ & $0.159 \pm 0.03$ & $0.153 \pm 0.03$ & 0.41 \\
\hline Tb.N (/mm) & $2.15 \pm 0.21$ & $2.22 \pm 0.24$ & 0.20 \\
\hline Tb.Th (mm) & $0.074 \pm 0.01$ & $0.069 \pm 0.01$ & 0.07 \\
\hline Tb.Sp (mm) & $0.394(0.37 ; 0.41)$ & $0.374(0.35 ; 0.41)$ & 0.46 \\
\hline Cortical area $\left(\mathrm{cm}^{2}\right)$ & $7.73 \pm 1.72$ & $7.75 \pm 1.41$ & 0.94 \\
\hline Ct.Th $(\mathrm{mm})$ & $0.889 \pm 0.22$ & $0.888 \pm 0.16$ & 0.99 \\
\hline Tibia geometry & $(n=33)$ & $(n=34)$ & \\
\hline BV/TV (1) & $0.167 \pm 0.02$ & $0.157 \pm 0.02$ & 0.11 \\
\hline Tb.N (/mm) & $2.13 \pm 0.26$ & $2.10 \pm 0.26$ & 0.56 \\
\hline Tb.Th (mm) & $0.078 \pm 0.01$ & $0.075 \pm 0.01$ & 0.12 \\
\hline $\mathrm{Tb} . \mathrm{Sp}(\mathrm{mm})$ & $0.399(0.34 ; 0.44)$ & $0.397(0.36 ; 0.44)$ & 0.54 \\
\hline Cortical area $\left(\mathrm{cm}^{2}\right)$ & $17.30 \pm 2.91$ & $16.60 \pm 2.49$ & 0.30 \\
\hline Ct.Th $(\mathrm{mm})$ & $1.458 \pm 0.30$ & $1.397 \pm 0.26$ & 0.36 \\
\hline
\end{tabular}

The $P$ values were calculated by unpaired Student's $t$-test or the Mann-Whitney rank sum test where appropriate. Significant results are highlighted in bold. VAT low, $_{\text {, visceral adipose tissue volume below median VAT; VAT }}$ high, visceral adipose tissue volume above median VAT; vBMD, volumetric bone mineral density; vBMD $_{\text {trab, }}$ trabecular vBMD; CSA, cross-sectional area; CSMI, cross-sectional moment of inertia; BV/TV, bone volume/tissue volume; Tb.N, trabecular number; Tb.Th, trabecular thickness; Tb.Sp, trabecular space; Ct.Th, cortical thickness.

At LS, vBMD trab was non-significantly lower in the $\mathrm{VAT}_{\text {high }}$ group compared with the $\mathrm{VAT}_{\text {low }}$ group $(P=0.22)$. DXA-derived aBMD at FN was significantly lower in the $\mathrm{VAT}_{\text {high }}$ group compared with the $\mathrm{VAT}_{\text {low }}$ group $(P=0.03)$, while no difference could be detected at LS $(P=0.96)$.

\section{HRpQCT-derived bone parameters across visceral fat median}

HRpQCT-derived bone parameters revealed no significant differences between VAT groups in any structural parameters in peripheral bone (Table 2).

\section{Bone biochemistry across visceral fat median}

Multivariate $t$-test on bone biochemistry across VAT groups failed to show overall significant effects $(P=0.60)$, thus markers of bone turnover, calcium metabolism, and sex hormone status did not differ between VAT groups. Table 3 presents the uncorrected $P$ values.

\section{Distribution of fat and sex hormones across $E_{2}$ median}

In order to investigate the effect of $\mathrm{E}_{2}$ on bone, the population was divided into two groups for comparison 
Table 3 Bone-relevant biochemical markers across visceral fat median. Data are expressed as mean \pm s.D. or median with interquartile range $(25 \% ; 75 \%)$.

\begin{tabular}{|c|c|c|c|}
\hline & VAT $_{\text {low }}(n=36)$ & $\operatorname{VAT}_{\text {high }}(n=36)$ & $P$ value \\
\hline BAP (U/I) & $28.6 \pm 7.6$ & $28.8 \pm 6.7$ & 0.91 \\
\hline Osteocalcin $(\mu \mathrm{g} / \mathrm{l})$ & $21.6(17.9 ; 26.2)$ & $20.1(16.3 ; 24.6)$ & 0.22 \\
\hline P1NP $(\mu \mathrm{g} / \mathrm{l})$ & $48.4 \pm 14.8$ & $43.0 \pm 12.3$ & 0.10 \\
\hline OPG (pmol/l) & $4.8 \pm 1.6$ & $4.7 \pm 1.4$ & 0.86 \\
\hline CTx $(\mathrm{ng} / \mathrm{ml})$ & $0.32(0 . \overline{2} 6 ; 0.44)$ & $0.30(0.23 ; 0.45)$ & 0.53 \\
\hline NTx (nmol/l) & $19.0 \pm 4.3$ & $18.7 \pm 4.5$ & 0.76 \\
\hline PTH (pmol/l) & $4.64(3 . \overline{7} 8 ; 6.60)$ & $5.11(4 . \overline{3} 8 ; 6.01)$ & 0.18 \\
\hline $\begin{array}{l}\text { 25-Hydroxy } \\
\text { vitamin D (nmol/l) }\end{array}$ & $47.3(28.6 ; 75.2)$ & $45.2(30.6 ; 65.4)$ & 0.82 \\
\hline Estradiol (pmol/l) & $36.3(14.5 ; 68.6)$ & $50.5(31.3 ; 75.0)$ & 0.18 \\
\hline $\begin{array}{l}\text { Testosterone } \\
(\mathrm{nmol} / \mathrm{l})\end{array}$ & $11.4 \pm 3.2$ & $11.5 \pm 3.3$ & 0.91 \\
\hline SHBG (nmol/l) & $31.4 \pm 9.9$ & $32.5 \pm 11.3$ & 0.66 \\
\hline
\end{tabular}

The $P$ values were calculated by unpaired Student's $t$-test or the Mann-

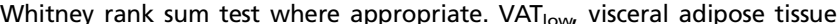

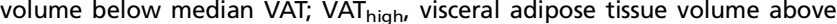
median VAT; BAP, bone alkaline phosphatase; P1NP, procollagen $1 \mathrm{~N}$-terminal propeptide; OPG, osteoprotegerin; CTx, C-terminal telopeptide of type 1 collagen; NTx, cross-linked N-terminal telopeptide of type 1 collagen; PTH, parathyroid hormone; SHBG, sex hormone-binding globulin.

based on $\mathrm{E}_{2}$ median $\left(\mathrm{E}_{2 \text { low }}\right.$ and $\left.\mathrm{E}_{2 \mathrm{high}}\right)$. Median $\mathrm{E}_{2}$ for $\mathrm{E}_{2 \text { low }}$ and $\mathrm{E}_{2 \text { high }}$ were 20.0 and $70.5 \mathrm{pmol} / 1$ respectively $(P<0.001)$. In addition, estrone was different between $\mathrm{E}_{2}$ groups (Table 4). Testosterone and androstenedione, which are converted by aromatase to $\mathrm{E}_{2}$ and estrone, respectively, and SHBG and insulin were comparable between groups (Table 4). Clinical characteristics such as age, weight, BMI, and WC did not differ between groups, nor did measures of whole-body fat mass, VAT, and SAT (Table 4).

\section{Bone density and structural parameters across $\mathrm{E}_{2}$ median}

At the lumbar spine, $\mathrm{vBMD}_{\text {trab }}$ was significantly lower in the $\mathrm{E}_{2 \text { low }}$ group compared with the $\mathrm{E}_{2 \text { high }}$ group $(P=0.04)$. Multivariate $t$-test on all QCT-derived hip parameters failed to show overall significant effects of $E_{2}$ at the hip $(P=0.25)$. The uncorrected $P$ values are presented in Table 5.

DXA-derived aBMD at FN was significantly lower in the $\mathrm{E}_{2 \text { low }}$ group compared with the $\mathrm{E}_{2 \text { high }}$ group $(P=0.02)$, and a similar yet only borderline significant difference was found at LS $(P=0.058)$.

\section{HRpQCT-derived bone parameters across $E_{2}$ median}

BV/TV and Tb.N were significantly lower and Tb.Sp significantly higher in the $\mathrm{E}_{2 \text { low }}$ group compared with the $\mathrm{E}_{2 \text { high }}$ group at both distal radius and distal tibia (Table 5).

\section{Bone biochemistry across $\mathrm{E}_{2}$ median}

Multivariate $t$-test on bone biochemistry across $\mathrm{E}_{2}$ groups failed to show overall significant effects $(P=0.16)$, thus markers of bone turnover and vitamin D levels did not differ between groups (Table 6).

\section{Impact of testosterone on bone parameters}

Thirty-six percent of the study population had testosterone deficiency with a testosterone level below $10.4 \mathrm{nmol} / 1$ $(8.0 \pm 1.6 \mathrm{nmol} / \mathrm{l})$. The remaining $64 \%$ of the population

Table 4 Clinical features, fat distribution, and sex hormones across estradiol median. Data are expressed as mean \pm s.D. or median with interquartile range $(25 \% ; 75 \%)$.

\begin{tabular}{|c|c|c|c|}
\hline & $\mathbf{E}_{\mathbf{2} \text { low }}(n=36)$ & $\mathbf{E}_{\mathbf{2} \text { high }}(n=36)$ & $P$ value \\
\hline Estradiol (pmol/l) & $20.0(12.1 ; 33.8)$ & $70.5(57.6 ; 93.5)$ & $<0.001$ \\
\hline Estrone $(p m o l / l)$ & $60.0(47.0 ; 80.3)$ & $94.0(66.3 ; 126.5)$ & $<0.001$ \\
\hline Testosterone $(\mathrm{nmol} / \mathrm{l})$ & $10.9 \pm 3.0$ & $11.9 \pm 3.4$ & 0.22 \\
\hline Androstenedione $(\mathrm{nmol} / \mathrm{l})$ & $1.95(1.38 ; 2.48)$ & $1.71(1.44 ; 2.14)$ & 0.45 \\
\hline SHBG $(\mathrm{nmol} / \mathrm{l})$ & $30.6 \pm 10.1$ & $33.3 \pm 10.9$ & 0.28 \\
\hline Insulin (pmol/l) & $81.3(52.2 ; 111.1)$ & $98.2(64.9 ; 113.9)$ & 0.30 \\
\hline Age (years) & $49.0 \pm 6.6$ & $49.4 \pm 6.1$ & 0.76 \\
\hline Weight (kg) & $107.1 \pm 13.6$ & $110.7 \pm 10.9$ & 0.21 \\
\hline BMI $\left(\mathrm{kg} / \mathrm{m}^{2}\right)$ & $33.1 \pm 3.3$ & $34.1 \pm 3.2$ & 0.21 \\
\hline Waist circumference $(\mathrm{cm})$ & $113(104 ; 120)$ & 115 (110; 118) & 0.26 \\
\hline Whole-body fat mass $(\mathrm{kg})$ & $32.6(28.6 ; 38.9)$ & 33.3 (31.9; 37.6) & 0.31 \\
\hline Visceral fat $\left(\mathrm{cm}^{3}\right)$ & $2638(2141 ; 3186)$ & $2824(2277 ; 3372)$ & 0.24 \\
\hline Subcutaneous fat $\left(\mathrm{cm}^{3}\right)$ & $5437(4471 ; 7119)$ & $5786(4589 ; 7222)$ & 0.54 \\
\hline
\end{tabular}

The $P$ values were calculated by unpaired Student's $t$-test or the Mann-Whitney rank sum test where appropriate. Significant results are highlighted in bold. $E_{2}$ low, estradiol below median $E_{2} ; E_{2}$ high, estradiol above median $E_{2} ;$ SHBG, sex hormone-binding globulin. 
Table 5 Bone characteristics across estradiol median. Data are expressed as mean \pm s.D. or median with interquartile range $(25 \% ; 75 \%)$.

\begin{tabular}{|c|c|c|c|}
\hline & $\mathbf{E}_{\mathbf{2} \text { low }}(n=36)$ & $\mathbf{E}_{\mathbf{2} \text { high }}(n=36)$ & $P$ value \\
\hline Lumbar spine $v B M D\left(\mathrm{~g} / \mathrm{cm}^{3}\right)$ & $0.137 \pm 0.03$ & $0.152 \pm 0.03$ & 0.04 \\
\hline \multicolumn{4}{|l|}{ Hip vBMD $\left(\mathrm{g} / \mathrm{cm}^{3}\right)$} \\
\hline \multicolumn{4}{|l|}{ Total hip } \\
\hline $\mathrm{VBMD}_{\text {integral }}$ & $0.307 \pm 0.05$ & $0.327 \pm 0.04$ & 0.07 \\
\hline $\mathrm{vBMD}_{\text {trab }}$ & $0.159 \pm 0.02$ & $0.168 \pm 0.02$ & 0.07 \\
\hline $\mathrm{vBMD}_{\text {cortical }}$ & $0.830 \pm 0.04$ & $0.815 \pm 0.03$ & 0.10 \\
\hline \multicolumn{4}{|l|}{ Femoral neck } \\
\hline $\mathrm{vBMD}_{\text {integral }}$ & $0.294 \pm 0.05$ & $0.304 \pm 0.04$ & 0.34 \\
\hline $\mathrm{vBMD}_{\text {trab }}$ & $0.179 \pm 0.03$ & $0.190 \pm 0.02$ & 0.07 \\
\hline $\mathrm{VBMD}_{\text {cortical }}$ & $0.605 \pm 0.04$ & $0.597 \pm 0.04$ & 0.34 \\
\hline \multicolumn{4}{|l|}{ Trochanter } \\
\hline $\mathrm{vBMD}_{\text {integral }}$ & $0.234 \pm 0.04$ & $0.247 \pm 0.03$ & 0.12 \\
\hline vBMD $_{\text {trab }}$ & $0.155 \pm 0.02$ & $0.162 \pm 0.02$ & 0.16 \\
\hline vBMD $_{\text {cortical }}$ & $0.770(0.73 ; 0.83)$ & $0.755(0.71 ; 0.78)$ & 0.04 \\
\hline \multicolumn{4}{|l|}{ Intertrochanteric } \\
\hline vBMD $_{\text {integral }}$ & $0.356 \pm 0.05$ & $0.385 \pm 0.06$ & 0.03 \\
\hline $\mathrm{vBMD}_{\text {trab }}$ & $0.162 \pm 0.03$ & $0.172 \pm 0.02$ & 0.11 \\
\hline $\mathrm{VBMD}_{\text {cortical }}$ & $0.840(0.82 ; 0.86)$ & $0.840(0.81 ; 0.86)$ & 0.73 \\
\hline \multicolumn{4}{|l|}{$\begin{array}{l}\text { Femoral neck geometry and } \\
\text { strength }\end{array}$} \\
\hline $\mathrm{CSA}_{\text {total }}\left(\mathrm{cm}^{2}\right)$ & $11.64 \pm 1.60$ & $12.02 \pm 1.27$ & 0.27 \\
\hline $\mathrm{CSA}_{\text {cortical }}\left(\mathrm{cm}^{2}\right)$ & $3.08(2.50 ; 3.32)$ & $3.15(2.78 ; 3.69)$ & 0.20 \\
\hline Cortical thickness $(\mathrm{mm})$ & $0.272(0.22 ; 0.32)$ & $0.281(0.24 ; 0.33)$ & 0.37 \\
\hline $\operatorname{CSMI}\left(\mathrm{mm}^{4}\right)$ & $6.80 \pm 1.67$ & $7.22 \pm 1.47$ & 0.26 \\
\hline Buckling ratio (1) & $7.58 \pm 2.44$ & $7.08 \pm 1.80$ & 0.33 \\
\hline \multicolumn{4}{|l|}{ Radius geometry } \\
\hline $\mathrm{BV} / \mathrm{TV}(1)$ & $0.149 \pm 0.03$ & $0.163 \pm 0.03$ & 0.04 \\
\hline Tb.N (/mm) & $2.12 \pm 0.24$ & $2.25 \pm 0.20$ & 0.02 \\
\hline Tb.Th (mm) & $0.070 \pm 0.01$ & $0.073 \pm 0.01$ & 0.37 \\
\hline Tb.Sp (mm) & $0.398(0.36 ; 0.44)$ & $0.374(0.35 ; 0.41)$ & 0.04 \\
\hline Cortical area $\left(\mathrm{cm}^{2}\right)$ & $7.63 \pm 1.69$ & $7.85 \pm 1.43$ & 0.57 \\
\hline Ct.Th $(\mathrm{mm})$ & $0.871 \pm 0.20$ & $0.906 \pm 0.20$ & 0.44 \\
\hline \multicolumn{4}{|l|}{ Tibia geometry } \\
\hline BV/TV (1) & $0.155 \pm 0.03$ & $0.169 \pm 0.02$ & 0.02 \\
\hline Tb.N (/mm) & $2.04 \pm 0.27$ & $2.20 \pm 0.22$ & 0.01 \\
\hline Tb.Th (mm) & $0.076 \pm 0.01$ & $0.077 \pm 0.01$ & 0.62 \\
\hline Tb.Sp (mm) & $0.406(0.37 ; 0.47)$ & $0.380(0.34 ; 0.42)$ & 0.02 \\
\hline Cortical area $\left(\mathrm{cm}^{2}\right)$ & $16.87 \pm 2.90$ & $17.02 \pm 2.54$ & 0.83 \\
\hline Ct.Th $(\mathrm{mm})$ & $1.416 \pm 0.29$ & $1.439 \pm 0.26$ & 0.73 \\
\hline
\end{tabular}

The $P$ values were calculated by unpaired Student's $t$-test or the Mann-Whitney rank sum test where appropriate. Significant results are highlighted in bold. $E_{2 \text { low, }}$ estradiol below median $E_{2} ; E_{2}$ high, estradiol above median $E_{2} ; \mathrm{VBMD}$, volumetric bone mineral density; $V B M D_{\text {trab, }}$ trabecular $\mathrm{VBMD}$; $C S A$, cross-sectional area; CSMI, cross-sectional moment of inertia; BV/TV, bone volume/tissue volume; Tb.N, trabecular number; Tb.Th, trabecular thickness; Tb.Sp, trabecular space; Ct.Th, cortical thickness.

had a relatively low mean testosterone level of $13.3 \pm$ $2.1 \mathrm{nmol} / 1$. Testosterone below $\left(\mathrm{T}_{\text {low }}\right.$ ) and above population mean $\left(\mathrm{T}_{\text {high }}\right)$ did not influence lumbar spine vBMD $_{\text {trab }}$ or hip vBMD $D_{\text {integral }}$ (neither TH nor site-specific). In addition, the structural parameters in peripheral bone were not significantly different between testosterone groups (data not shown).

\section{Impact of lean body mass on bone parameters}

Lean body mass below (lean low $_{\text {) }}$ and above population mean $\left(\right.$ lean $\left._{\text {high }}\right)$ did not affect lumbar spine $\mathrm{vBMD}_{\text {trab }}$ or hip $\mathrm{vBMD}_{\text {integral }}$ (neither $\mathrm{TH}$ nor site-specific). The structural parameters in radius were not significantly different between lean mass groups either. However, men with Lean $_{\text {high }}$ had significantly higher tibia BV/TV, Tb.N, and lower Tb.Sp (data not shown).

\section{Relation between effects of VAT and $E_{2}$ at the hip}

Multiple linear regression analysis revealed that VAT volume and $\mathrm{E}_{2}$ levels were significantly and independently associated with THvBMD integral $_{\text {(VAT: standardized }}$ $\beta=-0.316, P=0.007$ and $\mathrm{E}_{2}$ : standardized $\beta=0.261$, 
Table 6 Bone-relevant biochemical markers across estradiol median. Data are expressed as mean \pm s.D. or median with interquartile range $(25 \% ; 75 \%)$.

\begin{tabular}{|c|c|c|c|}
\hline & $\mathbf{E}_{2 \text { low }}(n=36)$ & $\mathbf{E}_{\mathbf{2} \text { high }}(n=36)$ & $P$ value \\
\hline BAP (U/I) & $28.2 \pm 7.5$ & $29.1 \pm 6.8$ & 0.60 \\
\hline Osteocalcin $(\mu \mathrm{g} / \mathrm{l})$ & $20.6(16.3 ; 26.8)$ & $20.5(18.0 ; 24.2)$ & 0.56 \\
\hline P1NP $(\mu \mathrm{g} / \mathrm{l})$ & $41.3(29.6 ; 58.8)$ & $46.3(39.4 ; 53.6)$ & 0.16 \\
\hline OPG (pmol/l) & $4.6 \pm 1.4$ & $4.9 \pm 1.6$ & 0.43 \\
\hline CTx $(\mathrm{ng} / \mathrm{ml})$ & $0.30(0.24 ; 0.51)$ & $0.32(0.27 ; 0.41)$ & 0.97 \\
\hline NTx (nmol/l) & $18.7 \pm 4.9$ & $18.9 \pm 3.8$ & 0.84 \\
\hline PTH (pmol/l) & $4.75(4.00 ; 5.68)$ & $5.75(4.22 ; 6.81)$ & 0.07 \\
\hline $\begin{array}{l}\text { 25-Hydroxy vitamin } \\
\text { D (nmol/l) }\end{array}$ & $48.7(34.5 ; 72.7)$ & $40.2(28.6 ; 69.0)$ & 0.22 \\
\hline
\end{tabular}

The $P$ values were calculated by unpaired Student's $t$-test or the MannWhitney rank sum test where appropriate. $E_{2 \text { low, }}$ estradiol below median $E_{2} ; E_{2 h i g h}$ estradiol above median $E_{2} ; B A P$, bone alkaline phosphatase; P1NP, procollagen $1 \mathrm{~N}$-terminal propeptide; OPG, osteoprotegerin; CTX, C-terminal telopeptide of type 1 collagen; NTx, cross-linked N-terminal telopeptide of type 1 collagen; PTH, parathyroid hormone.

$P=0.029)$, whereas testosterone and lean body mass were not associated with THvBMD integral $_{\text {(testosterone: } P=0.87}$ and lean mass: $P=0.70)$. Results were unchanged when the metabolic markers (IL6, HDL, and ALT) were included in the regression analysis. Then, the population was grouped according to VAT volume and $\mathrm{E}_{2}$ levels into four groups $\left(\mathrm{E}_{2 \text { low }}+\mathrm{VAT}_{\text {high }}(n=16), \mathrm{E}_{2 \text { low }}+\mathrm{VAT}_{\text {low }}(n=20)\right.$, $\mathrm{E}_{2}$ high $+\operatorname{VAT}_{\text {high }}(n=20)$, and $\left.\mathrm{E}_{2 \text { high }}+\operatorname{VAT}_{\text {low }}(n=16)\right)$. Integral vBMD at $\mathrm{TH}$ differed between groups (ANOVA overall $P=0.011$ ) and post hoc testing showed significantly lower $\mathrm{TH} \mathrm{vBMD}_{\text {integral }}$ in the $\mathrm{E}_{2 \text { low }}+\mathrm{VAT}_{\text {high }}$ group compared with the $\mathrm{E}_{2 \text { high }}+\mathrm{VAT}_{\text {low }}$ group $(P=0.006)$ (Fig. 2A). FN $T$-score was significantly lower in the $\mathrm{E}_{2 \text { low }}+\mathrm{VAT}_{\text {high }}$ group compared with the $\mathrm{E}_{2 \text { high }}+\mathrm{VAT}_{\text {low }}$ group $(-1.04 \pm 0.78$ vs $-0.13 \pm 0.70, P=0.004)$, and the prevalence of osteopenia was 50.0 and $6.3 \%$ respectively (Fig. 2B).

\section{Discussion}

In this cross-sectional study of 72 middle-aged obese men with MetS, we found that men with VAT volume above population median had lower integral and trabecular vBMD at the hip, and lower Ct.Th and higher buckling ratio at $\mathrm{FN}$, indicating a more fragile bone. $\mathrm{E}_{2}$ levels seemed more important at lumbar spine and the peripheral bones, as men with $\mathrm{E}_{2}$ below population median had lower lumbar spine $\mathrm{vBMD}_{\mathrm{trab}}$, reduced $\mathrm{BV} / \mathrm{TV}$ and Tb.N, and increased Tb.Sp at tibia and radius, while effects were less pronounced at the hip.
This population was very homogeneous and no correlations between VAT and BMI or WC were found. This allows us to interpret data without adjustments for $\mathrm{BMI}$ or WC that may confound results due to collinearity. Other studies have shown correlations between VAT and BMI or WC $(40,41)$, which is probably due to inclusion of populations with a large variability in age and BMI. Participants in our study all had relatively high amounts of VAT regardless of their BMI and WC, which explains the lack of correlation. Dividing the study subjects in two groups based on population-median VAT yielded no differences in age, BMI, WC, or SAT between groups, confirming that the impaired bone-specific outcomes observed in the $\mathrm{VAT}_{\text {high }}$ group are not biased by these characteristics. The use of QCT and HRpQCT provides measures of not only bone density, but also bone geometry and strength. The QCT technique reduces noise from excess soft tissue (42), which is why QCTderived vBMD may predict bone strength better in this category of patients, compared with DXA $(43,44)$. At the hip, QCT estimates integral bone density, trabecular bone density, and cortical bone density. The latter should be cautiously interpreted as cortex is very thin, and the resolution in scans with $3 \mathrm{~mm}$ slice thickness is suboptimal when discriminating cortical and trabecular compartments $(45,46)$. We found lower integral and trabecular vBMD at all measured hip sites, and lower Ct.Th and higher buckling ratio at $\mathrm{FN}$ in the $\mathrm{VAT}_{\text {high }}$ group. Furthermore, vBMD ${ }_{\text {cortical }}$ was significantly higher in the $\mathrm{VAT}_{\text {high }}$ group at $\mathrm{TH}$ and IT region. vBMD $_{\text {cortical }}$ primarily

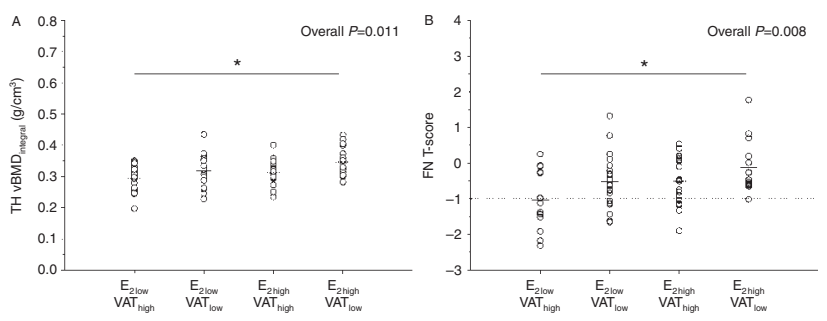

\section{Figure 2}

Total hip integral volumetric BMD (A) and femoral neck $T$-score (B) across combined $E_{2}$ and VAT groups ( $E_{2 \text { low }}+V_{\text {VAT }}$ high $(n=16)$, $\mathrm{E}_{2 \text { low }}+\operatorname{VAT}_{\text {low }}(n=20), \mathrm{E}_{2 \text { high }}+\mathrm{VAT}_{\text {high }}(n=20)$, and $\mathrm{E}_{2 \text { high }}+$ $\left.\operatorname{VAT}_{\text {low }}(n=16)\right)$. Dashed line distinguishes men with normal BMD (FN T-score $>-1$ ) and men with osteopenia (FN T-score $\leq-1$ and $>-2.5$ ). Open circle represents each trial subject and solid line represents group mean. The overall $P$ values were calculated by one-way ANOVA. ${ }^{*} P<0.01$. 
depends on cortical mineralization and porosity, without taking cortical area into account. However, bone size also determines resistance to load, and therefore CSA was measured at FN (34). FN CSA total was comparable between groups, while the $\mathrm{VAT}_{\text {high }}$ group had lower $\mathrm{CSA}_{\text {cortical }}$ and Ct.Th, indicating that VAT promotes cortical thinning at the FN, primarily due to endocortical bone loss. This could be a result of excess endocortical remodeling leading to transition of cortical bone to trabecular bone and a change in edge detection when analyzing the QCT scans. We speculate that the endocortical zone was detected as trabecular bone compartment in the $\mathrm{VAT}_{\text {high }}$ group, and would therefore expect the trabecular vBMD to be slightly overestimated. Indeed, we found a significantly lower trabecular bone density in the $\mathrm{VAT}_{\text {high }}$ group. Thus, the true trabecular bone density may be even lower than our results indicate.

We also investigated the effects of $\mathrm{E}_{2}$ levels on bone. Dividing the study subjects based on populationmedian $\mathrm{E}_{2}$ yielded no between-group differences in age, fat distribution, testosterone, androstenedione, or SHBG levels. This confirms that the impaired bone-specific outcomes we found in the $\mathrm{E}_{2 \text { low }}$ group are not biased by these characteristics. The $\mathrm{E}_{2 \text { low }}$ group was estrogen deficient with a median $\mathrm{E}_{2}$ of $20 \mathrm{pmol} / \mathrm{l}$ and the $\mathrm{E}_{2 \mathrm{high}}$ group was estrogen sufficient with a median $\mathrm{E}_{2}$ of $71 \mathrm{pmol} / \mathrm{l}$ (reference range for men between 20 and 70 years: $48-170 \mathrm{pmol} / \mathrm{l}$ (http://www.ssi.dk/English.aspx assessed 4th July 2014)). Men in the $\mathrm{E}_{2 \text { low }}$ group had lower lumbar spine trabecular bone density and impaired geometric measures at tibia and radius. Khosla et al. have previously described that rate of bone loss at the radius was associated with total and bioavailable $\mathrm{E}_{2}$ levels in elderly men but not in middle-aged men. Khosla et al. (47) also reported that bioavailable $\mathrm{E}_{2}$ levels below $40 \mathrm{pmol} / \mathrm{l}$ were associated with significantly higher rates of bone loss and higher levels of bone resorption markers in elderly men than bioavailable $\mathrm{E}_{2}$ levels above $40 \mathrm{pmol} / \mathrm{l}$. Although steroid levels are difficult to compare between laboratories, there is a striking difference between total $\mathrm{E}_{2}$ levels in our population and the population that Khosla et al. described. Median $\mathrm{E}_{2}$ level in this study was $43 \mathrm{pmol} / \mathrm{l}$ vs $103 \mathrm{pmol} / \mathrm{l}$ (middle-aged men) and $110 \mathrm{pmol} / 1$ (elderly men) described by Khosla et al. This lower level of $E_{2}$ in our population could explain why we, in contrary to Khosla et al., found that $\mathrm{E}_{2}$ is associated with bone density in middle-aged obese men. However, we did not find any differences in the bone resorption markers between groups, but the inter-individual variations in markers of bone resorption and bone formation are considerable.
The cross-sectional design may not be strong enough to demonstrate a possible difference.

Surprisingly, fat distribution was unaffected by $E_{2}$ level. We would have expected to find a positive association between levels of $\mathrm{E}_{2}$ and fat volume, due to increased aromatase activity $(19,20)$. Maggio et al. (48) investigated men with and without MetS and found higher $E_{2}$ levels in men with MetS. Our population had low levels of testosterone and as testosterone is the substrate for adipose tissue aromatase to produce $\mathrm{E}_{2}$, low testosterone levels could explain why adipose tissue volume did not affect $\mathrm{E}_{2}$ levels.

Multiple linear regression analysis revealed that VAT volume and $\mathrm{E}_{2}$ levels were independently associated with $\mathrm{THvBMD}_{\text {integral. }}$. Results presented in Fig. 2A clearly suggest an additive negative effect of VAT and low $\mathrm{E}_{2}$ levels on bone in men. To ensure that these findings are relevant in a clinical perspective, we investigated the FN $T$-scores across groups. Interestingly, we found a clinically relevant difference of more than 1 in FN $T$-scores between the $\mathrm{E}_{2 \text { low }}+\mathrm{VAT}_{\text {high }}$ group compared with the $\mathrm{E}_{2 \text { high }}+\mathrm{VAT}_{\text {low }}$ group. Comparing osteopenia prevalence in the $\mathrm{E}_{2 \text { low }}+$ $\mathrm{VAT}_{\text {high }}$ group $(50.0 \%)$ with the $\mathrm{E}_{2 \text { high }}+\mathrm{VAT}_{\text {low }}$ group $(6.3 \%)$, the clinical importance of VAT and $\mathrm{E}_{2}$ levels certainly becomes evident. Nielson et al. (49) reported a prevalence of osteopenia of $11.9 \%$ in a population of Caucasian men aged between 45 and 74 years with a BMI of $30+$. In comparison, our population comprises Caucasian men, average age 49 years (between 30 and 60 years) and average BMI $34 \mathrm{~kg} / \mathrm{m}^{2}$. The prevalence of osteopenia in our population is higher (29.2\%), which could be explained by the fact that all participants had MetS.

Testosterone and lean body mass did not influence BMD at the spine or hip in our population. However, lean body mass positively affected structural parameters at the tibia. A recent study on 40 younger women and ten men with MetS has demonstrated that lean mass was positively associated with HRpQCT-derived bone parameters at both tibia and radius (50). Unfortunately, they did not perform QCT at the hip or spine and did not have the opportunity to investigate effects of VAT and SAT separately.

Strengths of this study are the homogeneous population allowing us to interpret data without adjustments for BMI, WC, and testosterone levels, in addition to the use of several distinct methods to characterize bone. The limitations to this study are: first, the cross-sectional design does not allow us to determine causality. The differences we found in bone parameters across VAT and $\mathrm{E}_{2}$ groups could be caused by an overall worse metabolic status. However, when including metabolic markers in a 
multiple linear regression analysis, VAT and $\mathrm{E}_{2}$ are still the only markers significantly associated with THvBMD integral. Secondly, we included a limited number of subjects in this cohort. This study grounds on baseline data from another study (27) in which the sample size was determined. Post hoc power calculations suggested more than 90\% power to detect a $10 \%$ difference between groups in $\mathrm{TH}$ $\mathrm{vBMD}_{\text {integral }}$. However, the risk of type II errors is relatively high in relation to the biochemical bone turnover markers, as the inter-individual variation is considerable. This may explain the lack of difference in bone turnover markers in this study.

In summary, we found that an increased amount of VAT in obese middle-aged men with MetS is associated with harmful effects on BMD, bone geometry, and bone strength at the hip, whereas the harmful effects of low estrogen levels primarily unfolds at the lumbar spine, radius, and tibia. In addition, we found that VAT volume and $\mathrm{E}_{2}$ levels affect bone density at the hip independently and additively. The reduction in BMD in men with high VAT and low $\mathrm{E}_{2}$ has a magnitude implying clinical importance for future fracture risk in men suffering from MetS.

\section{Declaration of interest}

The authors declare that there is no conflict of interest that could be perceived as prejudicing the impartiality of the research reported.

\section{Funding}

This work is part of the research program LIRMOI research center (www.LIRMOI.com), which is supported by the Danish Council for Strategic Research (grant number 10-0934999). The HRPQCT scanner was supported by the Toyota Foundation, the Karen Elise Jensen Foundation, the AP Møller Maersk Foundation, and the Danish Osteoporosis Association.

\section{Acknowledgements}

The authors thank laboratory technicians Pia Hornbek, Lenette Pedersen, Lotte Sørensen, Lisbeth Flyvbjerg, and Tove Stenum, and radiographer Lissy F Kristiansen for their excellent technical assistance. Furthermore, they thank Lars Rejnmark and Jesper $S$ Thomsen for fruitful discussions of QCT measurements, and, finally, Jannie D Hald and Louise Wamberg for great helpfulness regarding HRPQCT and MRI respectively.

\section{References}

1 Wang YC, McPherson K, Marsh T, Gortmaker SL \& Brown M. Health and economic burden of the projected obesity trends in the USA and the UK. Lancet 2011378 815-825. (doi:10.1016/S0140-6736 (11)60814-3)

2 Leslie WD \& Morin SN. Osteoporosis epidemiology 2013: implications for diagnosis, risk assessment, and treatment. Current Opinion in Rheumatology 201426 440-446. (doi:10.1097/BOR. $0000000000000064)$

3 Lloyd JT, Alley DE, Hawkes WG, Hochberg MC, Waldstein SR \& Orwig DL. Body mass index is positively associated with bone mineral density in US older adults. Archives of Osteoporosis 20149175. (doi:10.1007/s11657-014-0175-2)

4 Reid IR, Ames R, Evans MC, Sharpe S, Gamble G, France JT, Lim TM $\&$ Cundy TF. Determinants of total body and regional bone mineral density in normal postmenopausal women - a key role for fat mass. Journal of Clinical Endocrinology and Metabolism $1992 \mathbf{7 5}$ 45-51. (doi:10.1210/jcem.75.1.1619030)

5 Felson DT, Zhang Y, Hannan MT \& Anderson JJ. Effects of weight and body mass index on bone mineral density in men and women: the Framingham study. Journal of Bone and Mineral Research 1993 8 567-573. (doi:10.1002/jbmr.5650080507)

6 Compston JE, Watts NB, Chapurlat R, Cooper C, Boonen S, Greenspan S, Pfeilschifter J, Silverman S, Diez-Perez A, Lindsay R et al. Obesity is not protective against fracture in postmenopausal women: GLOW. American Journal of Medicine 2011124 1043-1050. (doi:10.1016/j.amjmed.2011.06.013)

7 Nielson CM, Marshall LM, Adams AL, LeBlanc ES, Cawthon PM, Ensrud K, Stefanick ML, Barrett-Connor E \& Orwoll ES. BMI and fracture risk in older men: the osteoporotic fractures in men study (MrOS). Journal of Bone and Mineral Research 201126 496-502. (doi:10.1002/ jbmr.235)

8 Premaor MO, Pilbrow L, Tonkin C, Parker RA \& Compston J. Obesity and fractures in postmenopausal women. Journal of Bone and Mineral Research 201025 292-297. (doi:10.1359/jbmr.091004)

9 Vestergaard P. Discrepancies in bone mineral density and fracture risk in patients with type 1 and type 2 diabetes - a meta-analysis. Osteoporosis International 200718 427-444. (doi:10.1007/s00198-0060253-4)

10 de Liefde II, van der Klift M, de Laet CE, van Daele PL, Hofman A \& Pols HA. Bone mineral density and fracture risk in type- 2 diabetes mellitus: the Rotterdam study. Osteoporosis International 200516 1713-1720. (doi:10.1007/s00198-005-1909-1)

11 Schwartz AV, Sellmeyer DE, Ensrud KE, Cauley JA, Tabor HK, Schreiner PJ, Jamal SA, Black DM \& Cummings SR. Older women with diabetes have an increased risk of fracture: a prospective study. Journal of Clinical Endocrinology and Metabolism 2001 86 32-38. (doi:10.1210/jcem.86.1.7139)

12 Despres JP, Lemieux I \& Prud'homme D. Treatment of obesity: need to focus on high risk abdominally obese patients. BMJ 2001322 716-720. (doi:10.1136/bmj.322.7288.716)

13 Kopp HP, Kopp CW, Festa A, Krzyzanowska K, Kriwanek S, Minar E, Roka R \& Schernthaner G. Impact of weight loss on inflammatory proteins and their association with the insulin resistance syndrome in morbidly obese patients. Arteriosclerosis, Thrombosis, and Vascular Biology 200323 1042-1047. (doi:10.1161/01.ATV.0000073313. 16135.21)

14 Fontana L, Eagon JC, Trujillo ME, Scherer PE \& Klein S. Visceral fat adipokine secretion is associated with systemic inflammation in obese humans. Diabetes 200756 1010-1013. (doi:10.2337/ db06-1656)

15 Schett G. Effects of inflammatory and anti-inflammatory cytokines on the bone. European Journal of Clinical Investigation 201141 1361-1366. (doi:10.1111/j.1365-2362.2011.02545.x)

16 Fried SK, Bunkin DA \& Greenberg AS. Omental and subcutaneous adipose tissues of obese subjects release interleukin-6: depot difference and regulation by glucocorticoid. Journal of Clinical Endocrinology and Metabolism 199883 847-850. (doi:10.1210/jcem.83.3.4660)

17 Bruun JM, Lihn AS, Madan AK, Pedersen SB, Schiott KM, Fain JN \& Richelsen B. Higher production of IL-8 in visceral vs subcutaneous adipose tissue. Implication of nonadipose cells in adipose tissue. American Journal of Physiology. Endocrinology and Metabolism 2004286 E8-E13. (doi:10.1152/ajpendo.00269.2003) 
18 Cohen PG. Obesity in men: the hypogonadal-estrogen receptor relationship and its effect on glucose homeostasis. Medical Hypotheses 200870 358-360. (doi:10.1016/j.mehy.2007.05.020)

19 Schneider G, Kirschner MA, Berkowitz R \& Ertel NH. Increased estrogen production in obese men. Journal of Clinical Endocrinology and Metabolism 197948 633-638. (doi:10.1210/jcem-48-4-633)

20 Falahati-Nini A, Riggs BL, Atkinson EJ, O'Fallon WM, Eastell R \& Khosla S. Relative contributions of testosterone and estrogen in regulating bone resorption and formation in normal elderly men. Journal of Clinical Investigation 2000106 1553-1560. (doi:10.1172/ JCI10942)

21 Khosla S, Melton LJ III, Robb RA, Camp JJ, Atkinson EJ, Oberg AL, Rouleau PA \& Riggs BL. Relationship of volumetric BMD and structural parameters at different skeletal sites to sex steroid levels in men. Journal of Bone and Mineral Research 200520 730-740. (doi:10.1359/ JBMR.041228)

22 Gilsanz V, Chalfant J, Mo AO, Lee DC, Dorey FJ \& Mittelman SD. Reciprocal relations of subcutaneous and visceral fat to bone structure and strength. Journal of Clinical Endocrinology and Metabolism 200994 3387-3393. (doi:10.1210/jc.2008-2422)

23 Yamaguchi T, Kanazawa I, Yamamoto M, Kurioka S, Yamauchi M, Yano $\mathrm{S} \&$ Sugimoto T. Associations between components of the metabolic syndrome versus bone mineral density and vertebral fractures in patients with type 2 diabetes. Bone 200945 174-179. (doi:10.1016/j.bone.2009.05.003)

24 Choi HS, Kim KJ, Kim KM, Hur NW, Rhee Y, Han DS, Lee EJ \& Lim SK. Relationship between visceral adiposity and bone mineral density in Korean adults. Calcified Tissue International 201087 218-225. (doi:10.1007/s00223-010-9398-4)

25 Kim HI, Kim JT, Yu SH, Kwak SH, Jang HC, Park KS, Kim SY, Lee HK \& Cho YM. Gender differences in diagnostic values of visceral fat area and waist circumference for predicting metabolic syndrome in Koreans. Journal of Korean Medical Science 201126 906-913. (doi:10.3346/jkms. 2011.26.7.906)

26 Abate N, Garg A, Peshock RM, Stray-Gundersen J \& Grundy SM. Relationships of generalized and regional adiposity to insulin sensitivity in men. Journal of Clinical Investigation 199596 88-98. (doi:10.1172/JCI118083)

27 Ornstrup MJ, Harslof T, Kjaer TN, Langdahl BL \& Pedersen SB. Resveratrol increases bone mineral density and bone alkaline phosphatase in obese men: a randomized placebo-controlled trial. Journal of Clinical Endocrinology and Metabolism 201499 4720-4729. (doi:10.1210/ jc.2014-2799)

28 Alberti KG, Zimmet P \& Shaw J. Metabolic syndrome - a new worldwide definition. A Consensus Statement from the International Diabetes Federation. Diabetic Medicine 200623 469-480. (doi:10.1111/ j.1464-5491.2006.01858.x)

29 Positano V, Gastaldelli A, Sironi AM, Santarelli MF, Lombardi M \& Landini L. An accurate and robust method for unsupervised assessment of abdominal fat by MRI. Journal of Magnetic Resonance Imaging 200420 684-689. (doi:10.1002/jmri.20167)

30 Arif H, Racette SB, Villareal DT, Holloszy JO \& Weiss EP. Comparison of methods for assessing abdominal adipose tissue from magnetic resonance images. Obesity 200715 2240-2244. (doi:10.1038/oby. 2007.266)

31 Abrahamsen B, Gram J, Hansen TB \& Beck-Nielsen H. Cross calibration of QDR-2000 and QDR-1000 dual-energy X-ray densitometers for bone mineral and soft-tissue measurements. Bone 199516 385-390. (doi:10.1016/8756-3282(94)00054-9)

32 Mosekilde L, Beck-Nielsen H, Sorensen OH, Nielsen SP, Charles P, Vestergaard P, Hermann AP, Gram J, Hansen TB, Abrahamsen B et al. Hormonal replacement therapy reduces forearm fracture incidence in recent postmenopausal women - results of the Danish Osteoporosis Prevention Study. Maturitas 200036 181-193. (doi:10.1016/S03785122(00)00158-4)
33 Borggrefe J, Graeff C, Nickelsen TN, Marin F \& Gluer CC. Quantitative computed tomographic assessment of the effects of 24 months of teriparatide treatment on 3D femoral neck bone distribution, geometry, and bone strength: results from the EUROFORS study. Journal of Bone and Mineral Research 201025 472-481. (doi:10.1359/ jbmr.090820)

34 Bouxsein ML. Determinants of skeletal fragility. Best Practice \& Research. Clinical Rheumatology 200519 897-911. (doi:10.1016/j.berh. 2005.07.004)

35 Rivadeneira F, Zillikens MC, de Laet CE, Hofman A, Uitterlinden AG, Beck TJ \& Pols HA. Femoral neck BMD is a strong predictor of hip fracture susceptibility in elderly men and women because it detects cortical bone instability: the Rotterdam study. Journal of Bone and Mineral Research 200722 1781-1790. (doi:10.1359/jbmr. 070712)

36 Boutroy S, Bouxsein ML, Munoz F \& Delmas PD. In vivo assessment of trabecular bone microarchitecture by high-resolution peripheral quantitative computed tomography. Journal of Clinical Endocrinology and Metabolism 200590 6508-6515. (doi:10.1210/jc.2005-1258)

37 Hansen S, Hauge EM, Beck Jensen JE \& Brixen K. Differing effects of PTH 1-34, PTH 1-84, and zoledronic acid on bone microarchitecture and estimated strength in postmenopausal women with osteoporosis: an 18-month open-labeled observational study using HR-pQCT. Journal of Bone and Mineral Research 201328 736-745. (doi:10.1002/ jbmr.1784)

38 Burghardt AJ, Link TM \& Majumdar S. High-resolution computed tomography for clinical imaging of bone microarchitecture. Clinical Orthopaedics and Related Research 2011469 2179-2193. (doi:10.1007/ s11999-010-1766-x)

39 Poulsen MM, Vestergaard PF, Clasen BF, Radko Y, Christensen LP, Stodkilde-Jorgensen H, Moller N, Jessen N, Pedersen SB \& Jorgensen JO. High-dose resveratrol supplementation in obese men: an investigatorinitiated, randomized, placebo-controlled clinical trial of substrate metabolism, insulin sensitivity, and body composition. Diabetes 2013 62 1186-1195. (doi:10.2337/db12-0975)

40 Janssen I, Heymsfield SB, Allison DB, Kotler DP \& Ross R. Body mass index and waist circumference independently contribute to the prediction of nonabdominal, abdominal subcutaneous, and visceral fat. American Journal of Clinical Nutrition 200275 683-688.

41 Carroll JF, Chiapa AL, Rodriquez M, Phelps DR, Cardarelli KM, Vishwanatha JK, Bae S \& Cardarelli R. Visceral fat, waist circumference, and BMI: impact of race/ethnicity. Obesity 200816 600-607. (doi:10.1038/oby.2007.92)

42 Khoo BC, Brown K, Cann C, Zhu K, Henzell S, Low V, Gustafsson S, Price RI \& Prince RL. Comparison of QCT-derived and DXA-derived areal bone mineral density and $T$ scores. Osteoporosis International 2009 20 1539-1545. (doi:10.1007/s00198-008-0820-y)

$43 \mathrm{Yu}$ EW, Thomas BJ, Brown JK \& Finkelstein JS. Simulated increases in body fat and errors in bone mineral density measurements by DXA and QCT. Journal of Bone and Mineral Research 201227 119-124. (doi:10.1002/jbmr.506)

44 Yu EW, Bouxsein ML, Roy AE, Baldwin C, Cange A, Neer RM, Kaplan LM \& Finkelstein JS. Bone loss after bariatric surgery: discordant results between DXA and QCT bone density. Journal of Bone and Mineral Research 201429 542-550. (doi:10.1002/jbmr.2063)

45 Zebaze R, Ghasem-Zadeh A, Mbala A \& Seeman E. A new method of segmentation of compact-appearing, transitional and trabecular compartments and quantification of cortical porosity from high resolution peripheral quantitative computed tomographic images. Bone 201354 8-20. (doi:10.1016/j.bone.2013.01.007)

46 Lang TF, Li J, Harris ST \& Genant HK. Assessment of vertebral bone mineral density using volumetric quantitative CT. Journal of Computer Assisted Tomography 199923 130-137. (doi:10.1097/00004728199901000-00027)

47 Khosla S, Melton LJ III, Atkinson EJ \& O'Fallon WM. Relationship of serum sex steroid levels to longitudinal changes in bone 
density in young versus elderly men. Journal of Clinical Endocrinology and Metabolism 200186 3555-3561. (doi:10.1210/ jcem.86.8.7736)

48 Maggio M, Lauretani F, Ceda GP, Bandinelli S, Basaria S, Paolisso G, Giumelli C, Luci M, Najjar SS, Metter EJ et al. Estradiol and metabolic syndrome in older Italian men: the InCHIANTI study. Journal of Andrology 201031 155-162. (doi:10.2164/jandrol.108. 006098)
49 Nielson CM, Srikanth P \& Orwoll ES. Obesity and fracture in men and women: an epidemiologic perspective. Journal of Bone and Mineral Research 201227 1-10. (doi:10.1002/jbmr.1486)

50 Madeira E, Mafort TT, Madeira M, Guedes EP, Moreira RO, de Mendonca LM, Lima IC, de Pinho PR, Lopes AJ \& Farias ML. Lean mass as a predictor of bone density and microarchitecture in adult obese individuals with metabolic syndrome. Bone 201459 89-92. (doi:10.1016/j.bone.2013.11.004)

Received 16 September 2014

Revised version received 10 November 2014

Accepted 21 November 2014 